\title{
Diagnóstico de miofibrossarcoma de alto grau em cão - relato de caso
}

\author{
Diagnosis of high-degree myofibrosarcoma in dog - case report \\ Diagnóstico de miofibrosarcoma de alto grado em perro - reporte de caso
}

Recebido: 25/10/2021 | Revisado: 02/11/2021 | Aceito: 10/11/2021 | Publicado: 17/11/2021

\author{
Mayara Lima Kavasaki \\ ORCID: https://orcid.org/0000-0001-6206-039X \\ Faculdade Marechal Rondon, Brasil \\ Universidade Federal de Mato Grosso, Brasil \\ E-mail: mayarakawa@gmail.com \\ Mateus Tomazelli Barboza \\ ORCID: https://orcid.org/0000-0002-1718-9052 \\ Faculdade Marechal Rondon, Brasil \\ E-mail: mateustbarboza7@gmail.com \\ Julia Almodóvar Stein \\ ORCID: https://orcid.org/0000-0002-7581-5912 \\ Faculdade Marechal Rondon, Brasil \\ E-mail: juliaalmodovarstein@hotmail.com \\ Giancarlo Rieger \\ ORCID: https://orcid.org/0000-0001-5801-6134 \\ Faculdade Marechal Rondon, Brasil \\ Universidade Cesumar, Brasil \\ Universidade Brasil, Brasil \\ E-mail: giancarlo.rieger@ faron.edu.br \\ Leandro da Silva Rocha \\ ORCID: https://orcid.org/0000-0002-1318-8589 \\ Universidade Federal de Mato Grosso, Brasil \\ E-mail: leandrorochavet@gmail.com \\ Ariane de Oliveira Troguilho \\ ORCID: https://orcid.org/0000-0001-9495-0151 \\ Faculdade Marechal Rondon, Brasil \\ Centro universitário Luterano de Ji-Paraná, Brasil \\ E-mail: Ariane.troguilho@gmail.com
}

\begin{abstract}
Resumo
Os Sarcomas de tecidos moles (STM) na oncologia veterinária, compreendem um grupo de neoplasias mesenquimais malignas, com acometimento principalmente da pele e subcutâneo, possuem margens mal definidas com altos índices de recidiva local e moderado potencial metastático. O diagnóstico de rotina é realizado com base no histórico do animal, sinais clínicos, porém a confirmação é realizada através de exames citológicos e histopatológicos. Complementarmente a quimioterapia, radioterapia e remoção cirúrgica são utilizados para proporcionar uma maior sobrevida aos pacientes. O presente estudo tem por objetivo reportar um caso de Mixofibrossarcoma de Alto Grau, detectado através do exame imuno-histoquimico, uma variação do (STM), em um paciente canino, fila brasileiro, macho, com um ano de idade, atendido em uma clínica veterinária situada no município de Vilhena- Rondônia, apresentando inicialmente um nódulo em região da escápula direita, com evolução rápida e crescimento progressivo após remoção cirúrgica. Os tutores optaram pela eutanásia do animal.
\end{abstract}

Palavras-chave: Neoplasia; Sarcoma; Imuno-istoquímica; Cão.

\begin{abstract}
Soft tissue sarcomas (STM) in veterinary oncology comprise a group of malignant mesenchymal neoplasms, mainly affecting the skin and subcutaneous tissue, with ill-defined margins with high rates of local recurrence and moderate metastatic potential. Routine diagnosis is based on the animal's history, clinical signs, but confirmation is performed through cytological and histopathological exams. In addition to chemotherapy, radiotherapy and surgical removal are used to provide greater survival for patients. The present study aims to report a case of High-Grade Myxofibrosarcoma, detected by immunohistochemical examination, a variation of (STM), in a one-year-old male patient, Brazilian row, treated at a clinic veterinarian located in the city of Vilhena- Rondônia, initially presenting a nodule in the region of the right scapula, with rapid evolution and progressive growth after surgical removal. The guardians opted for the animal's euthanasia.
\end{abstract}

Keywords: Neoplasm; Sarcoma; Immunohistochemistry; Dog. 


\begin{abstract}
Resumen
Los sarcomas de tejidos blandos (STM) en oncología veterinaria comprenden un grupo de neoplasias mesenquimales malignas, que afectan principalmente a la piel y al tejido subcutáneo, con márgenes mal definidos con altas tasas de recurrencia local y potencial metastásico moderado. El diagnóstico de rutina se basa en la historia del animal, los signos clínicos, pero la confirmación se realiza mediante exámenes citológicos e histopatológicos. Además de la quimioterapia, la radioterapia y la extirpación quirúrgica se utilizan para proporcionar una mayor supervivencia a los pacientes. El presente estudio tiene como objetivo reportar un caso de Mixofibrosarcoma de Alto Grado, detectado por examen inmunohistoquímico, una variación de (STM), en un paciente masculino de un año, fila brasileña, atendido en una clínica veterinaria ubicada en la ciudad de Vilhena- Rondônia, presenta inicialmente un nódulo en la región de la escápula derecha, de rápida evolución y crecimiento progresivo tras la extirpación quirúrgica. Los guardianes optaron por la eutanasia del animal.
\end{abstract}

Palabras clave: Neoplasia; Sarcoma; Inmunohistoquímica; Perro.

\title{
1. Introdução
}

O cuidado com a saúde dos animais de companhia, especialmente de cães e gatos, tem crescido nos últimos anos, o cão passou a ser membro da família e não é mais considerado um simples animal de estimação. Nesse contexto, os problemas de pele passaram a chamar mais a atenção dos tutores. Fibrossarcomas são neoplasias malignas de fibroblastos que produzem tecido conjuntivo colágeno, não originam osso nem cartilagem.

Fibrossarcomas centrais originam-se do tecido fibroso dentro da cavidade medular, enquanto que fibrossarcomas periosteais se originam do tecido conjuntivo do periósteo. De forma geral, fibrossarcomas centrais crescem mais lentamente, são acompanhadas por menores formações de osso neoformado reativo, fazem metástase mais vagarosamente e produzem uma massa tecidual menor que os osteossarcomas (Carlton, 1998).

Os tumores denominados como sarcomas de tecidos moles (STM) são neoplasias mesenquimais de tecidos de origem mesodérmica (tecido fibroso, adiposo, músculos, cápsula sinovial, vasos sanguíneos e linfáticos) como também as neoplasias de revestimento de nervos periféricos, de origem ectodérmica (Gross et al., 2005). Os STM correspondem de 8 a $15 \%$ de todos os tumores cutâneos e subcutâneos caninos (Daleck; de Nardi, 2016).

O tumor pode ser encontrado em qualquer localização, entretanto, é particularmente encontrado no tecido subcutâneo (Moulton, 1990). Mais comumente encontrado em cães e gatos, podendo aparecer em qualquer espécie animal (Moulton, 1990).

Cerca de $20 \%$ dos pacientes com STM apresentam metástases ao diagnóstico. O desenvolvimento de metástases possui relação multifatorial, sendo a histologia, o grau de diferenciação, o tamanho e a localização das lesões os mais importantes. Lesões da região retroperitonial estão mais frequentemente associadas à disseminação metastática, devido provavelmente à sua maior vascularização e ao maior volume tumoral à época do diagnóstico (Enziger; Lattes; Torloni, 1969).

Múltiplos fatores influenciam na recorrência local, tendo especial importância o grau de diferenciação tumoral, que é o melhor indicador prognóstico isolado da eventual recidiva. O envolvimento de linfonodos e invasão vásculo-nervosa ou óssea, também são fatores de maior risco, principalmente nos pacientes tratados com cirurgia conservadora (Kindblom; Lodding; Angervall,1983)

O exame físico meticuloso tem vital importância na determinação do volume tumoral e sua eventual extensão ou fixação a ossos ou feixes vásculosnervosos, além da avaliação linfonodal locorregional, que apesar de pouco frequentemente acometida, tem incidência aumentada de até $30 \%$ em alguns subtipos histológicos, como rabdiomiossarcoma e leiomiossarcoma (Eljabbour; et al., 1990).

A avaliação pré-operatória deve ser realizada através de métodos de imagens, como raio-x, para se descartar presença de lesões metastáticas. Metástases hematogênicas ocorrem em geral nos pacientes com tumores de alto grau de malignidade, em geral nos dois primeiros anos do diagnóstico, necessitando uma avaliação rigorosa, tomografia computadorizada e ressonância nuclear magnética são úteis tanto para definição da localização como da extensão do tumor primário assim como 
para a determinação da presença de possíveis metástases a distância. Os sítios de disseminação mais frequentes são os ossos e o fígado, com cerca de 15 a 25\% de chance de serem acometidos (Dudgeon; Patrick, 1927; Eich; Whitehair; Morof; Heeb, 2000).

É necessário durante o tratamento estabelecer uma terapia adjuvante eficaz para sarcomas de tecidos moles (STSs), estudos demonstram que a administrado em combinação com quimioterapia à base de doxorrubicina, melhora a eficácia do tratamento (Tian; et al., 2021; Greto; et al.,2018).

Macroscopicamente, os fibrossarcomas são branco-acinzentados, preenchem parte da cavidade medular e substituem o osso esponjoso e o cortical (Carlton, 1998). Os que apresentam crescimento lento, frequentemente, possuem consistência um pouco firme e clara. Os que crescem rapidamente são encefalóides e de cor rósea. Necrose e hemorragias podem aparecer no seio dos tumores (Santos, 1988).

\section{Metodologia}

Um cão da raça Fila Brasileiro, com um ano de idade, pesando 41,6 kg, foi atendido apresentando apatia, apetite caprichoso e lesões ulceradas em região escapular e mucosa oral com aspecto de couve -flor. Durante a avaliação clínica o animal apresentava aumento de linfonodos subescapulares e retrofaríngeos. No exame físico da cavidade oral foi possível observar a presença de dois nódulos, ulcerados de aspecto friável, infiltrativo com característica pendular, localizados em mucosa oral interna da boca, comprometendo a área da mucosa gengival, tais nódulos apresentavam aproximadamente $4,0 \mathrm{~cm}$ cada.

Foram solicitados os seguintes exames laboratoriais: hemograma completo, proteínas totais e frações, creatinina, alanina aminotransferase (ALT), sorologia e teste imunocromatográfico para Leishmaniose, exame ultrassonográfico para avaliação de abdômen total, radiografia de crânio e tórax e exame histopatológico de dois fragmentos, sendo um de pele da região escapular e outro da cavidade oral.

Por meio das imagens radiográficas, no crânio foi possível observar aumento de volume dos tecidos moles ventrolateralmente ao terço rostromediano do ramo horizontal da mandíbula esquerda, sem evidências de alterações radiográficas em estruturas ósseas cranianas. $\mathrm{O}$ toráx se apresentava dentro da normalidade, sem evidências radiográficas de presença de nódulos metastáticos dispersos em parênquima pulmonar. Os resultados dos exames Ensaio Imunoenzimático e Imunocromatográfico para deteç̧ão de Leishmaniose $s p$ apresentaram-se não reagentes. Os exames hematológicos e bioquímicos encontravam-se dentro dos valores de referência.

Os fragmentos coletados para o exame histopatológico, foram divididos em amostra 1- Escápula: Fragmento acastanhado, medindo $1,0 \times 0,4 \times 0,3 \mathrm{~cm}$. Ao corte, apresentando superfície castanha esbranquiçada, com área enegrecida, macia por vezes friável e irregular e amostra 2- Cavidade Oral: Fragmento acastanhado, medindo 0,8 x 0,7 x 0,5cm. Ao corte, superfície esbranquiçada, macia por vezes friável e irregular. O Laudo apresentado como sendo o diagnóstico da amostra A Sarcoma de Partes Moles Grau II e amostra B - Sarcoma Fusocelular Pouco Diferenciado, Morfologicamente Sugestivo de Fibrosarcoma. Sendo que a amostra A continha Grau de diferenciação: pouco diferenciado; necrose tecidual: presente em mais de 50\% da área tumoral; nível de invasão/infiltração: difuso e índice mitótico: 10 campos/40x.

A seguir, na Figura 1 expõe elementos pertinentes para o presente estudo, onde o animal em questão apresenta, na orelha esquerda, um padrão hiperêmico difuso. Tal fator é de grande relevancia para o diagnóstico diferencial de neoplasias cutâneas. 
Figura 1 - Padrão Hiperêmico difuso na orelha do animal.

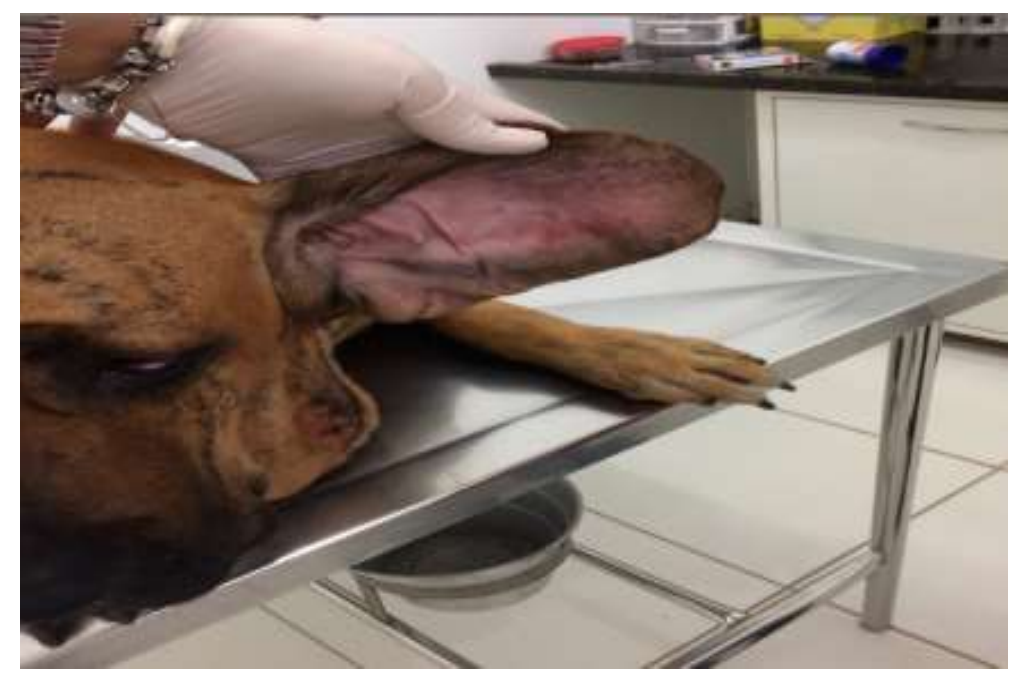

Fonte: Mayara Lima Kavasaki (2019).

Ademais, a Figura 2 tem como principal objetivo evidenciar o miofibrossarcoma de alto grau, que apresenta uma densa área de necrose em região cervical.

Figura 2 - Miofibrossarcoma de alto grau em região cervical.

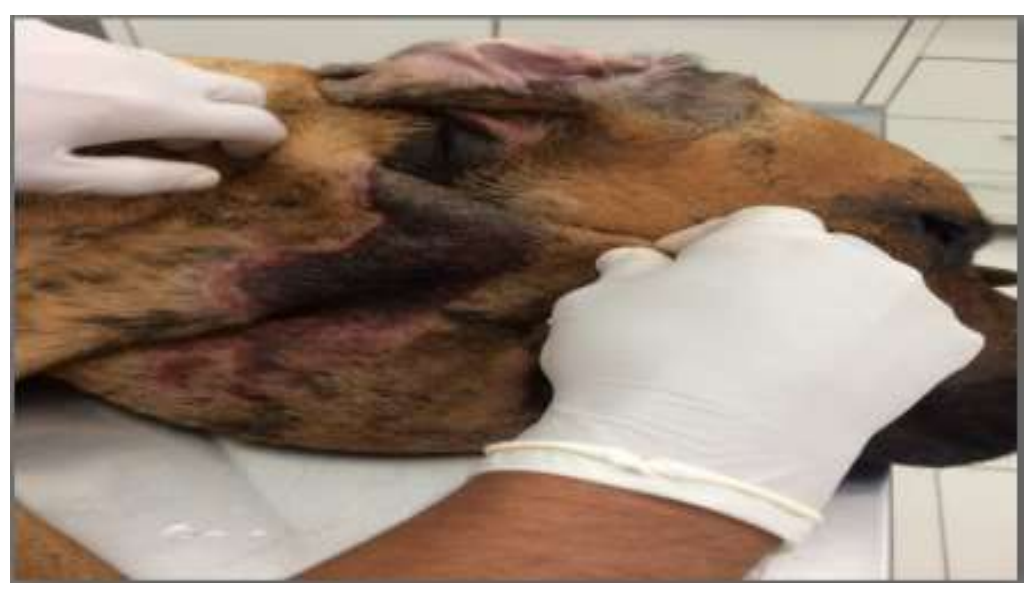

Fonte: Mayara Lima Kavasaki (2019).

A Figura 3 evidencia o resultado do exame histopatológico do animal, que expõe uma análise tecidual das lesões, com o objetivo de informar a gravidade, extensão e a intensidade da lesão. 
Figura 3 - Exame histopatológico.

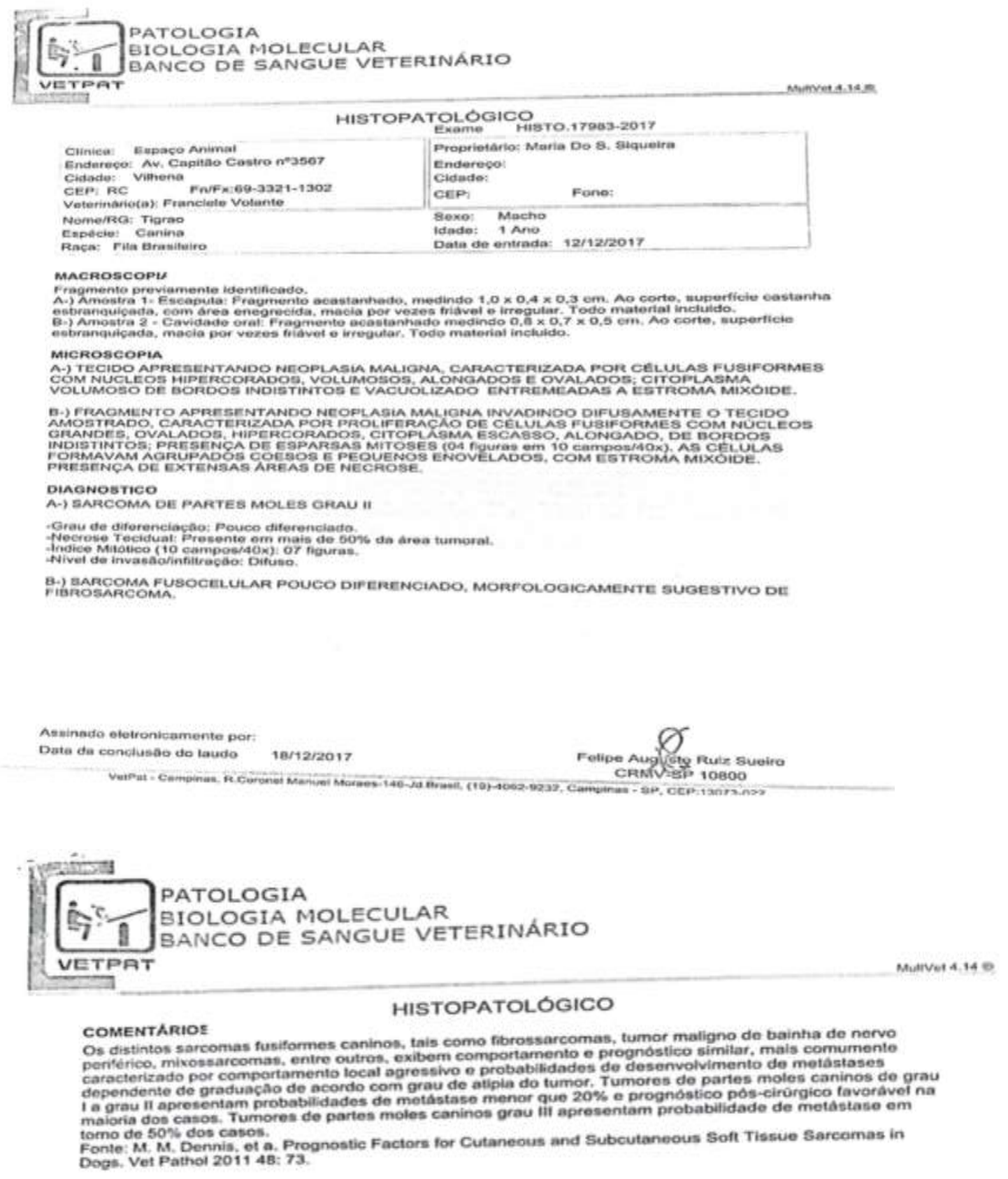

Fonte: Mayara Lima Kavasaki.

A Figura 4 mostra o exame imunoistoquímica, o qual permite a detecção de antígenos específicos e imunofenotipagem de tecidos ou agentes infecciosos, determinando o grau do sarcoma. 
Figura 4 - Exame imunohistoquímica.

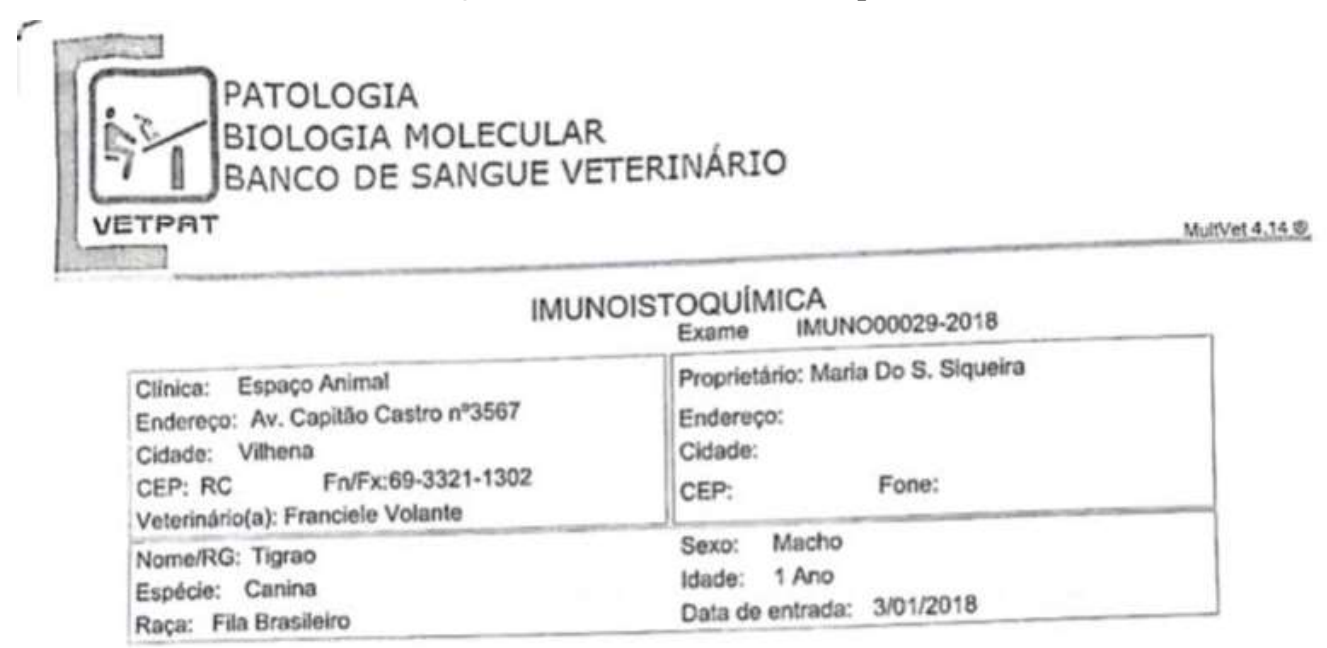

\section{MATERIAL}

Bloco histologico parafinado identificado como 17983-17

SARCOMA FUSOCELULAR POUCO DIFERENCIADO.

.Os

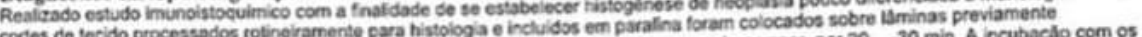

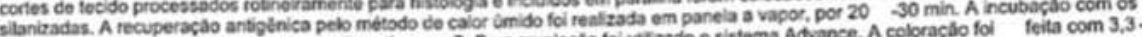

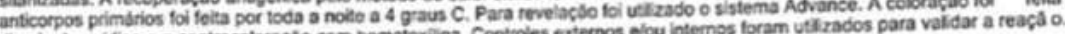

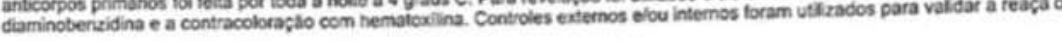

\section{IMUNOISTOQUIMICA}

As células neoplásicas imunocxpressarem: Desmina e Vimentina.

Não expressaram: S100; 1A4; HHF35; Cocktel Melanoma e GFAP.

Marcador de proliferaçắo Ki67 positivo em aproximadamente $50 \%$ das células neoplásicas.

\section{CONCLUSÃO: O PERFIL IMUNOISTOQUIMICO E MORFOLÓGICO FAVORECEM O} DIAGNÓSTICO DE MIOFIBROSARCOMA DE ALTO GRAU.

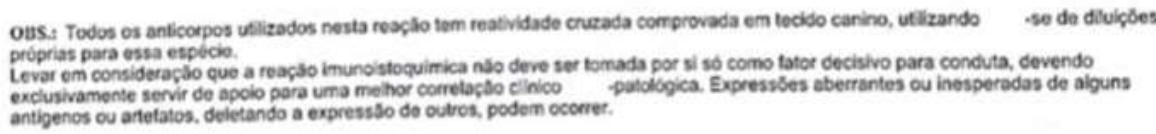

Assinado eletronicamente por:

Data da conclusăo do laudo

$11 / 01 / 2018$

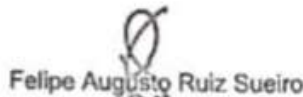

CRMVISP 10800

VetPat - Campinas, R. Coronel Manuel Moraos-146-Jd-Brasil, (19)-4062-9232, Campinas - SP. CEP:13073-022

Fonte: Mayara Lima Kavasaki (2019).

\section{Resultados e Discussão}

Durante o tempo decorrido para confirmação do diagnóstico o animal passou a apresentar prurido intenso em região afetada e a pele apresentava-se em padrão hiperêmico difuso (Figura 1 e 2).

No presente relato o diagnóstico histopatológico foi de sarcoma fusocelular, sugestivo de fibrossarcoma. É importante considerar a possibilidade de disseminação mesmo em tumores que possuem baixos índices metastáticos, obtendo um estadiamento clínico fidedigno, de forma a estabelecer a melhor conduta terapêutica para o paciente. 
Os fragmentos coletados para o exame histopatológico foram encaminhados para realização de imuno-histoquímica e conclui-se que o perfil imunohistoquímico e morfológico favoreciam o diagnóstico de miofibrosarcoma de alto grau.

Foi indicado o procedimento cirúrgico para ressecção da área afetada pela equipe cirúrgica, e através de uma decisão em conjunto o tutor optou pela eutanásia do animal.

Os sarcomas aparecem na forma de tumores mesenquimais malignos, que progridem lentamente e são altamente invasivos. Eles são o terceiro tipo de tumor que afeta cães grandes com idade média de oito anos, como Golden Retrievers e Dobermans. Os machos costumam ser mais suscetíveis (Mcentee, 2012; Gardner et al., 2013).

Durante o exame físico do animal relatado, não foi verificado outras anormalidades a não ser a presença do nódulo subcutâneo de consistência firme na superfície da pele. Ao estadiamento clínico do paciente, não foi encontrado nenhuma alteração.

A realização de estudos no que diz respeito à prevalência de determinada condição ou grupo de doenças é de extrema importância na medicina veterinária e na pesquisa científica como um todo, pois permitem que informações definitivas sejam acumuladas para uso futuro (Kaldrymidou et al., 2002), o presente trabalho descreve a ocorrência de tumor cutâneo encontrado em um cão adulto.

No cenário atual brasileiro ainda existem poucos estudos descrevendo a ocorrência de tumores cutâneos em cães (Machado et al., 1963; Souza et al., 2006; Bellei et al., 2006; Meirelles et al., 2010; Teixeira et al., 2010).

É importante ressaltar que dados clínicos no que dizem respeito à faixa etária, sexo e a raça dos animais também são informações epidemiológicas importantes que devem sempre estar presentes nas fichas e nos laudos fornecidos por hospitais e médicos veterinários.

A imuno-histoquímica vem sendo usada na rotina diagnóstica e na pesquisa em patologia humana, desde 1970 (Ruiz et al., 2005). Seu uso na medicina veterinária é mais recente, porém nos países desenvolvidos é uma técnica bastante difundida quando comparada aos países emergentes.

Os resultados do presente trabalho mostraram a importância do uso da imuno-histoquímica como ferramenta auxiliar para o patologista veterinário que faz o diagnóstico de tumores de pele, nesse sentido, o diagnóstico preciso permite ao oncologista maior acurácia na determinação do prognóstico e na escolha do tratamento para seus pacientes. Assim como na Medicina Humana, os tumores em pele e partes moles nos animais domésticos devem ser diagnosticados e tratados prontamente.

\section{Conclusão}

Conclui-se que a possibilidade de realização de procedimentos cirúrgicos, como no caso descrito seria necessário para garantir uma maior sobrevida ao animal. Além disso, reforça-se a importância da adesão de terapias adjuvantes por parte dos tutores visando atingir um sucesso adequado do tratamento do câncer.

A incidência de miofibrossarcomas em cães é rara, com comprometimento de pele, tecido subcutâneo da boca e nariz, cavidade oral, fascia e periósteo. Ressaltando assim a extrema importância de realizações de trabalhos futuros que possam auxiliar na clínica médica veterinária.

\section{Referências}

Robert, K. Y. \& Daniel, G. (2001) Estudo de caso: planejamento e métodos. 2.ed. https://saudeglobaldotorg1.files.wordpress.com/2014/02/yinmetodologia_da_pesquisa_estudo_de_caso_yin.pdf.

Bellei, M. H. M., Neves, D. S., \& Gava, A. (2006) Prevalence study of canine skin tumours in Santa Catarina, Brazil, between 1998 and $2002 ., 73-79$. 
Tian, Z., Wang, J., \& Yang, J. (2021) et al. Apatinib with doxorubicin and ifosfamide as neoadjuvant therapy for high-risk soft tissue sarcomas: a retrospective cohort study. Invest New Drugs 39, 1724-1731. https://doi.org/10.1007/s10637-021-01139-w

Bronden, L. B., Eriksen, T., \& Kristensen, A. T. (2010) Mast cell tumours and other skin neoplasia in Danish dogs.

Carlton, W. W., \& Mc Gavin, M. D. (1998) Patologia veterinária especial de Thomsom. 742-743.

Greto D, Loi M, \& Saieva C, (2018) et al. Safety of concurrent adjuvant radiotherapy and chemotherapy for locally advanced soft tissue sarcoma. Tumori Journal. 104(5):322-329. 10.1177/0300891618765565

Dudgeon, L. S., \& Patrick, C. V. (1927) A new method for the rapid microscopical diagnosis of tumors: with na account of 200 cases so examined. The British Journal of Surgery, p. 250-261.

Eich, C. S., Whitehair, J. G., Moroff, S. D., \& Heeb, L. A (2000) The accuracy of intraoperative cytopathological dianosis compared with conventional histopathological diagnosis. Journal of the American Animal Hospital Association, 36(1), 16-8.

El-jabbbour, J. N., Akhtar, S.S., Kerr, G. R., Mclaren, K. M., Smyth, A. \& Rodger F. C. Leonard. Prognostic Factors for Survival in Soft Tissue Sarcoma. Br. J. Cancer, 62, 857-61. 1990.

Enzinger, F.M., Lattes, R., \& Torloni, R. (1969) Histological typing of soft tissue tumors Internacional Histological Classification of Tumors. World Health Organization.

Gardner, D. G., \& Baker, D. C. (2013) Canine oral fibrosarcomas: a retrospective analysis of 65 cases (1998-2010). Veterinary and comparative oncology, 18 .

Ginn, P. E., Mansell, J. E. K. L., Rakich, P. M. Jubb, K. V. F., Kennedy, P. C., \& Palmer's, N. C. (2007) Pathology of domestic animals. 5 ed. Philadelphia: Saunders Elsevier. 1, Cap. 5, 553-781.

Goldschmidt, M. H., \& Hendrick, M. J. (2002) Tumors in domestic animals. (4a ed.) Ames: Iowa State, 2, 44-117.

Gross T. L., Ihrke P. J., Walder E. J., \& Affolter V. K. (2005) Skin Disease of the Dog and Cat, Clinical Histopathologic Diagnosis. (2nd Ed) Oxford. Blackwell Publishing, 616-626.

Jones, T.C., Hunt, R. D., \& King, N.W. (2000) Patologia veterinária. (4a ed.) Manole, 1053-1059.

Kaldrymidou, H., Leontides, L., \& Koutinas, A. F (2002). et al. Prevalence, distribution and factors associated with the presence and the potential for malignancy of cutaneous neoplasms in 174 dogs admitted to a clinic in northern Greece. J. Vet. Med. A. Physiol. Pathol. Clin. Med., 49(2), 87-91.

Kindblom, L.G., Lodding, P., \& Angervall, L. (1983) Clear cell sarcoma of tendons and aponeuroses. Virchows Arch. A, 401, 109-128.

Machado, A. V., Silva, J. M. L., \& Curial, O. (1963) Incidência de blastomas em animais no Brasil. Arq. Esc. Vet., 25, $327-401$.

Mcentee, M. C. (2012) Veterinary radiation therapy: review and current state of the the art. Oral and maxillofacial surgery in dogs and cats. Pennsylvania: Elsevier. 387-402.

Meirelles, A. E. W. B., Oliveira, E. C., \& Rodrigues, B. A. (2010) et al. Prevalência de neoplasmas cutâneos em cães da Região Metropolitana de Porto Alegre, RS: 1.017 casos (2002-2007). Pesq. Vet. Bras., 30(11), 968-973.

Moulton, J. E. Tumors in domestic animals. (1990) 3. ed. California: university of California press, 25-27.

Parkin D. M. (1992) Cancer incidence in five Continents. Iarc Scientific Publications, 120, 456.

Pakhrin, B., Kang, M. S., Bae, I. H. et al. (2007) Retrospective study of canine cutaneous tumors in Korea. J. Vet., 8(3), 229-236.

Ramos-vara, J. A., Kiupel, M., \& Baszler, T. (2008) et al. Suggested guidelines for immunohistochemical techniques in veterinary diagnostic laboratories. J. Vet. Diagn. Invest., 20(4), 393-413.

Rothman, K. (1986) Epidemiology.J.Modern Epidemiology.Brow and Company, 123, 478.

Ruiz, F. S., Alessi, A. C., \& Chagas, C. A. (2005) et al. Imuno-histoquímica na patologia veterinária diagnóstica: uma revisão crítica. J. Bras. Patol. Med. Lab., 41(4), 263-270.

Santos, J. A. (1988) Patologia geral dos animais domésticos. (3a ed.) Guanabara, 314-315.

Scott, D. W., Miller, W. H., \& Griffin, C. E. (2001) Muller \& Kirk. Small animal dermatology. (6a ed.) Saunders Company.

Souza, T. M., Fighera, R. A., \& Irigoyen, L. F. (2006) et al. Estudo retrospectivo de 761 tumores cutâneos em cães. Cienc. Rural., 36(2), 555-560.

Teixeira, T. F., da Silva, T. C., \& Cogliati, B. (2010) et al. Retrospective study of melanocytic neoplasms in dogs and cats. Braz. J. Vet. Pathol., 3(2), 100-104.

Vail D. M., \& Withrow S. J. Tumors of the Skin and Subcutaneous Tissues. (2001). Small Animal Clinical Oncology. (3a ed.) W. B. Saunders, 233-260.

Withrow, S. J, \& Macewen's, E. G. (2007) Small clinical veterinary oncology. Why worry about cancer in pets. (5a ed.) Saunders Elsevier.

Yager, J. A., \& Wilcock, B. P. (1994) Color atlas and text of surgical pathology of the dog and cat. London: Wolfe, 1, $291-292$.

Yager, J. A., \& Wilcock, B. P. (1994) Tumors of the skin and associated tissues Color atlas and text of surgical pathology of the dog and cat dermatopathology and skin tumors. Wolfe. 1, 239-31. 